\title{
Influence of the orthorhombic phase content on the dielectric and magnetic properties of $\mathrm{YMnO}_{3}$
}

\author{
M. Sánchez-Pérez ${ }^{1}$, O. J. Dura ${ }^{1,2, *}$, J. P. Andrés ${ }^{1,3}$, R. López Antón ${ }^{1,3}$, J. A. Gonzalez ${ }^{1,3}$ and M. A. \\ López de la Torre ${ }^{1,2}$ \\ ${ }^{1}$ Departamento de Física Aplicada, Universidad de Castilla-La Mancha, 13071 Ciudad Real, \\ Spain \\ ${ }^{2}$ Instituto de Investigaciones Energéticas y Aplicaciones Industriales (INEI), Universidad de \\ Castilla-La Mancha, 13071 Ciudad Real, Spain \\ ${ }^{3}$ Instituto Regional de Investigación Científica Aplicada (IRICA), Universidad de Castilla-La \\ Mancha, 13071 Ciudad Real, Spain \\ *Corresponding author \\ E-mail address: Oscar.juan@uclm.es
}

\section{Keywords}

Manganites

Impedance spectroscopy

Multiferroics

\begin{abstract}
Here we report on the dielectric and magnetic properties of polycrystalline samples of $\mathrm{YMnO}_{3}$ where the ratio between both orthorhombic (o-YMO) and hexagonal ( $h$-YMO) phases has been controlled by means of appropriate sintering treatments. Interestingly, we observe that the dielectric behavior is dominated by the presence of the metastable o-YMO phase, even though its amount remains as a minority phase. On the other hand, it also influences the magnetic behavior for sintering temperatures below $1000{ }^{\circ} \mathrm{C}$, and in addition, a weak ferromagnetic contribution is always detected in our polycrystalline samples regardless the o-YMO content.
\end{abstract}

\section{Introduction}

Multiferroic materials are defined as materials where more than one ferroic order coexist, usually, ferroelectric and magnetic order. They can be exploited in both electrical and magnetic functionalities, but their principal advantage is the possibility of switching the spontaneous magnetization by an applied electric field and vice versa. This interesting effect is due to the coupling between the ferroelectric and magnetic orders of the system, and it could be very useful for technological applications, such as sensors, magnetic data storage, non-volatile memory, transducers, magnetoelectronic devices, or spintronics. ${ }^{1-3}$. However, although the complex coupling between orbital, spin, charge and lattice degrees of freedom in perovskites gives rise to remarkable properties such as ferroelectricity or colossal magnetoresistance, there are few examples of multiferroic perovskites. Therefore, manganites such as $\mathrm{RMnO}_{3}(\mathrm{R}$ as rareearth element) have been classified as an important type of multiferroic materials ${ }^{4}$.

Depending on the size of the rare-earth element in the perovskite structure, $\mathrm{RMnO}_{3}$ can crystallize in two different structures: hexagonal for $\mathrm{R}=\mathrm{Ho}, \mathrm{Er}, \mathrm{Tm}, \mathrm{Yb}$, Lu or $\mathrm{Y}$, and orthorhombic for $\mathrm{R}=\mathrm{La}, \mathrm{Ce}, \mathrm{Pr}, \mathrm{Nd}, \mathrm{Sm}, \mathrm{Eu}, \mathrm{Gd}, \mathrm{Tb}$ or Dy. The yttrium manganite $\mathrm{YMnO}_{3}$ is a special case, because it can display both structures: the hexagonal one (h-YMO), which is the thermodynamically stable structure, and the orthorhombic one (o-YMO), which is a metastable 
phase stabilized by means of special synthesis methods, as mechanochemical synthesis, annealing at high pressures or synthesis at high temperatures ${ }^{1,4-6}$. It is noteworthy that the multiferroic order in both structures has different sources, as it is also the case for $\mathrm{HoMnO}_{3}{ }^{7,8}$. While hexagonal $\mathrm{YMnO}_{3}$ is a multiferroic type-I in which the source of ferroelectricity is not in the magnetic order, orthorhombic $\mathrm{YMnO}_{3}$ is type-II, where the ferroelectricity is induced by the magnetism of the system ${ }^{9}$. The hexagonal $\mathrm{h}-\mathrm{YMO}$ (space group $\mathrm{P} 6_{3} \mathrm{~cm}$ ) presents antiferromagnetic order with a Néel temperature $\mathrm{T}^{\mathrm{h}} \mathrm{N}$ about $70 \mathrm{~K}$ and ferroelectric order below a Curie temperature $T_{C} \simeq 920 \mathrm{~K}^{4,10-14}$. The significant difference between both transition temperatures also indicates the ferroelectric order does not have a magnetic origin. This structure consists of non-connected layers of $\mathrm{MnO}_{5}$ trigonal bipyramids, instead of classical octahedra in perovskite, separated by $\mathrm{Y}$ layers heaped up along the $\mathrm{c}$-axis ${ }^{15}$. These bipyramids are tilted with respect to the $\mathrm{c}$-axis, so the $\mathrm{Y}$ ions are unequally displaced in this direction. This displacement generates a spontaneous polarization in the c-axis $5,8,13,16,17$. In contrast, the antiferromagnetic order is due to the spin-interactions in the geometrically frustrated $\mathrm{Mn}$-spin system ${ }^{15,18}$. The $\mathrm{Mn}^{3+}-\mathrm{Mn}^{3+}$ interaction is the origin of the antiferromagnetic order type $\mathrm{A}$ of this phase, with high Curie-Weiss temperatures of about $-500 \mathrm{~K}^{10,15,17,19-24}$. The structural transition from hexagonal to orthorhombic phase gives rise to changes in the dielectric and magnetic properties, as well as different origin of ferroelectric and magnetic orders. The orthorhombic oYMO (space group Pnma) consists of polyhedral units built up by $\mathrm{Mn}^{3+}$ ions in the center of $\mathrm{MnO}_{6}$ octahedra, where $\mathrm{Mn}-\mathrm{O}$ bonds are relatively short and strong ${ }^{25-27}$. In this phase, both ferroelectric and magnetic orders are intimately related, as the former is induced by the latter, therefore giving rise to similar order temperatures. Hence, the antiferromagnetic order type $\mathrm{E}$ of this phase has a Néel temperature $\mathrm{T}^{\circ} \mathrm{N}$ of about $40 \mathrm{~K}$ whereas the ferroelectric transition is about $30 \mathrm{~K}^{1,4,5,28}$.

Multiferroic yttrium manganite compound $\left(\mathrm{YMnO}_{3}\right)$ has been intensively studied due to its remarkable properties, interesting for fundamental science as well as for its potential applications. With regard to the electrical properties, there are several works where the p-type conductivity in $\mathrm{YMnO}_{3}$ is discussed in terms of different sources showing inconsistent results between them: while some authors have related this $p$-type conductivity with a thermally activated small polaron hopping between localized $\mathrm{Mn}^{3+}$ and $\mathrm{Mn}^{4+}$ sites, others have pointed to interstitial oxygen or oxygen vacancies as a possible source of this conductivity in hexagonal $\mathrm{YMnO}_{3}{ }^{29-32}$. Previous works have reported on the electrical characterization by using impedance spectroscopy, but they are mainly focused on the characterization of the hexagonal phase and offer disagreeing interpretations ${ }^{16,20,33,34}$. As far as we know, there are no published results about the influence of the simultaneous presence of both $\mathrm{h}-\mathrm{YMO}$ and o-YMO phases on the dielectric properties in polycrystalline $\mathrm{YMnO}_{3}$. It results interesting to investigate the electricaltransport in the coexisting $\mathrm{h}-\mathrm{YMO}$ and o-YMO to provide useful information for nanocomposites or multiphase films which have potential applicability in magnetodielectric devices or domainwall-based electronics ${ }^{35,36}$. Therefore, in this work we analyze systematically for the first time the influence of the metastable orthorhombic phase on the electrical and magnetic properties of the hexagonal $\mathrm{YMnO}_{3}$. For this purpose, we have synthetized a family of samples where the amount of the orthorhombic phase has been tuned by selecting the appropriated sintering conditions.

\section{Materials and methods}

The reference compound is the hexagonal $\mathrm{YMnO}_{3}(\mathrm{~h}-\mathrm{YMO})$ and it was synthetized through solidstate reaction from $\mathrm{Y}_{2} \mathrm{O}_{3}$ and $\mathrm{Mn}_{2} \mathrm{O}_{3}$ starting powders ${ }^{33,37}$. The stoichiometric mixture of starting 
powders was pressed into one disk with a $40 \mathrm{~mm}$ diameter and sintered several times at 1100 ${ }^{\circ} \mathrm{C}$ after different grindings. Then, in order to obtain samples with a modified microstructure (nano/meso-structure) from the reference one obtained by the conventional solid-state route, part of the powder was ball milled during $48 \mathrm{~h}$ at $250 \mathrm{rpm}$ in zirconia ceramic jars using a ball to powder ratio (bpr) of 6:1. Through this method we obtained hexagonal $\mathrm{YMnO}_{3}$ with a grain size in the nanometer range. From this nanostructured $\mathrm{h}-\mathrm{YMO}$ powders, we were able to control the microstructure and the amount of orthorhombic $\mathrm{YMnO}_{3}$ phase (o-YMO) by means of different moderate heat treatments on the as-milled nanostructured powders, at temperatures between 700 and $1100{ }^{\circ} \mathrm{C}$ for 2 hours. This family of samples will be referred herein as YMO_700, YMO_800, YMO_900, YMO_1000 and YMO_1100 accordingly with the temperature of the treatment. The X-ray diffraction patterns at room temperature were obtained using a Phillips diffractometer model X'pert MPD ( $\mathrm{Cu} \mathrm{K} \alpha$ radiation; $\lambda=1.5406 \AA$ ) by scanning $2 \theta$ from $20^{\circ}$ to $100^{\circ}$ (figures only show data up to $55^{\circ}$ for a better comparison). High-Resolution Scanning Electronic Microscopy (HRSEM) images were performed using a ZEISS- GeminiSEM 500 operated at $2 \mathrm{kV}$ acceleration voltage. Simultaneous Differential Scanning Calorimetry and Thermogravimetric (DSC-TG) experiments were performed in a TGA-DSC Netzsch Jupiter STA $449 \mathrm{C}$ up to $1100{ }^{\circ} \mathrm{C}$ at $5 \mathrm{~K} / \mathrm{min}$ under synthetic air flow. The magnetic characterization was performed using an Evercool MPMS-XL SQUID magnetometer (Quantum Design). The ZeroField-Cooled (ZFC) and Field-Cooled (FC) curves were obtained under an external magnetic field of $\mathrm{H}=100$ Oe between $300 \mathrm{~K}$ and $5 \mathrm{~K}$. The hysteresis loops were measured at $5 \mathrm{~K}$ under applied magnetic fields between $-50 \mathrm{kOe}$ and $50 \mathrm{kOe}$, after a field cooling from room temperature in a magnetic field of $\mathrm{H}=10 \mathrm{kOe}$. The dielectric characterization was performed using an Agilent E4980A LCR Meter operating between $20 \mathrm{~Hz}$ and $1 \mathrm{MHz}$ in a temperature range from $320 \mathrm{~K}$ to $440 \mathrm{~K}$ using silver paste electrodes to obtain electrical contacts.

\section{Results and discussion}

\subsection{Structural characterization}

Figure 1 (left panel) shows the X-ray diffraction patterns for all the samples analyzed in this work and also for the as-milled nanostructured powders (labeled as a-m NP in the graph). In the case of YMO_1100 and YMO_1000 samples (as well as the initial powder), all reflections indexed correspond to the hexagonal structure $\mathrm{h}-\mathrm{YMO}$ and no traces of orthorhombic phase o-YMO are detected. Therefore, we can assure that these two samples are single h-YMO phase. On the other hand, in the case of YMO_900 and YMO_800 samples, reflections corresponding to both orthorhombic and hexagonal phases are present, which indicates coexistence of h-YMO and oYMO phases. Finally, for YMO_700 sample the main peaks observed correspond to h-YMO but at least two peaks corresponding to the o-YMO are also observed, indicating that small traces of that phase are present. By comparing these patterns, we could conclude that YMO_900 is the sample with maximum o-YMO phase content. In figure 1 (right panel), a quantitative Rietveld analysis corresponding to the YMO_900 sample is shown as an example. From this refinement we can conclude that the amount of each phase present in this sample is $70 \%$ and $30 \%$ for the hexagonal and the orthorhombic phase, respectively. The study of the YMO_800 sample refinement results in $73 \%$ and $27 \%$ for h-YMO and o-YMO, respectively, concluding YMO_900 is the sample with the highest o-YMO amount although o-YMO content in these both samples is similar.

In addition, it is noticeable the effect of the sintering treatment on the microstructure, see inset of figure 1 (left panel). Thus, as we expected there is a progressive narrowing of the diffraction peaks as temperature of treatment increases. This typical effect reflects the grain growth by 
sintering at increasing temperature. In figure 2 we present the HRSEM images of the family of samples. By comparing all the samples we observe an evolution of the microstructure as the narrowing of the diffraction peaks indicates, nevertheless it appears the microstructure doesn't show too large differences. The table included in figure 2 summarizes the average grain sizes estimations obtained by analyzing these images with the Gwyddion software ${ }^{38}$.
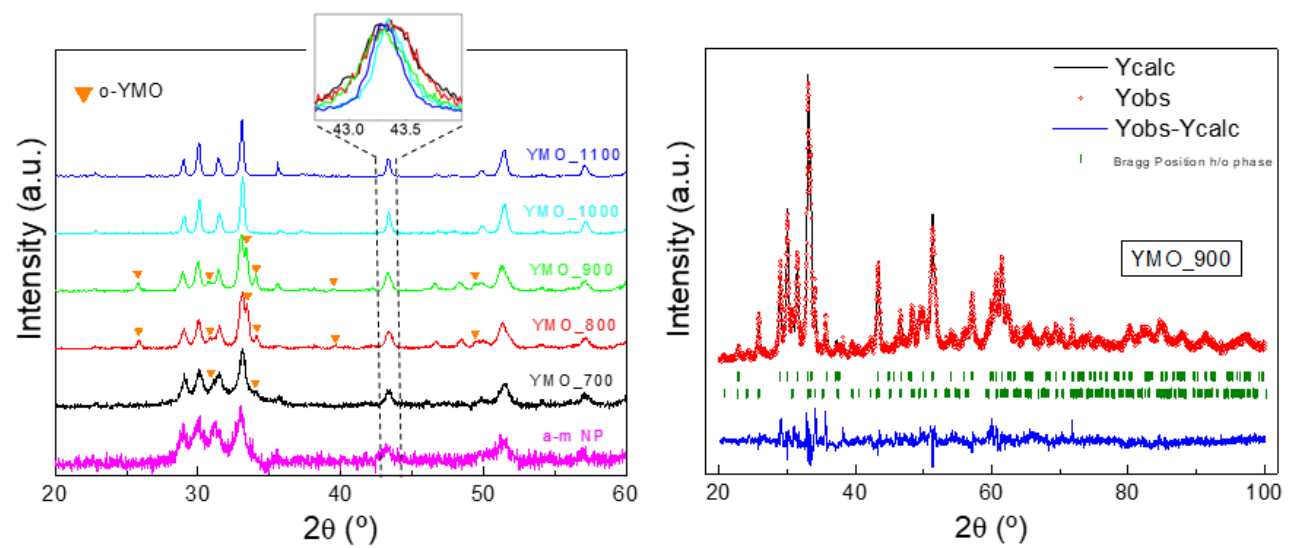

Figure 1. X-ray diffraction patterns of the family of samples studied in this work including the as-milled nanostructured powders (labelled as a-m NP) (left panel), the inset points the narrowing effect due to the grain growth as treatment temperature increases. The Rietveld refinement analysis of YMO_900 sample (right panel). 

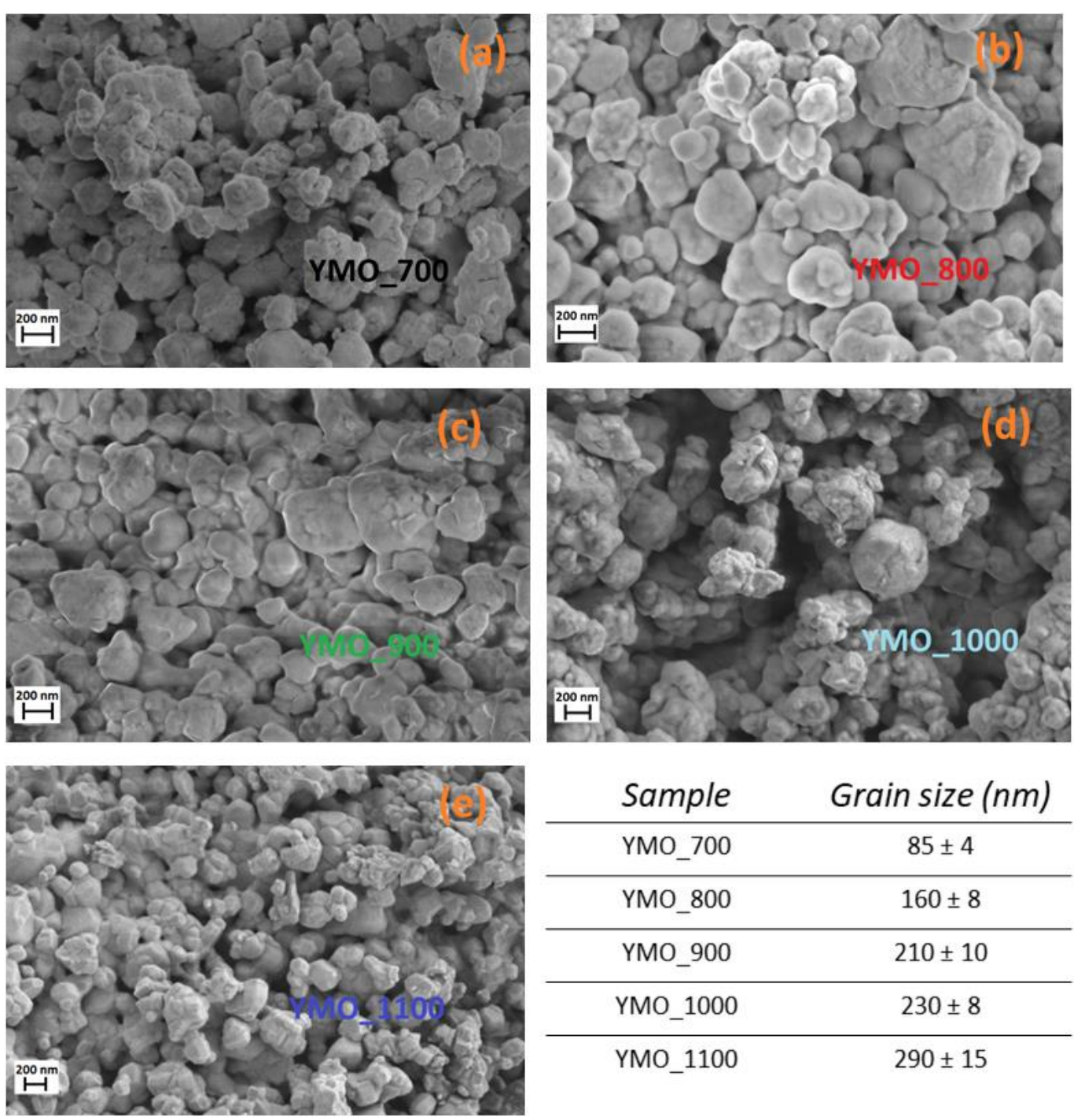

\begin{tabular}{cc} 
Sample & Grain size $(\mathrm{nm})$ \\
\hline YMO_700 & $85 \pm 4$ \\
\hline YMO_800 & $160 \pm 8$ \\
\hline YMO_900 & $210 \pm 10$ \\
\hline YMO_1000 & $230 \pm 8$ \\
\hline YMO_1100 & $290 \pm 15$
\end{tabular}

Figure 2. HRSEM images of the samples analysed: YMO_700 (a); YMO_800 (b); YMO_900 (c); YMO_1000 (d) and YMO_1100 (e). The inserted table summarizes the grain size estimations obtained from the analysis of HRSEM images.

Differential scanning calorimetry (DSC) and thermogravimetric curves (TG) for the as-milled nanostructured h-YMO were performed (figure 3), which are useful to verify the transition temperatures between both o-YMO and h-YMO phases. We can observe two significant peaks in the DSC curve: the first peak at $813^{\circ} \mathrm{C}$ corresponds to an endothermic phase transition which is accompanied of a slight loss of mass (less than $1 \%$ ), while the second peak is an exothermic phase transition at $918{ }^{\circ} \mathrm{C}$ without a significant loss of mass. Other authors have shown structural changes reporting a transition $\mathrm{h}-\mathrm{YMO} \rightarrow \mathrm{O}-\mathrm{YMO}$ at $\mathrm{T} \simeq 840^{\circ} \mathrm{C}$ and a transition o-YMO $\rightarrow \mathrm{h}-\mathrm{YMO}$ at $\mathrm{T} \simeq 960^{\circ} \mathrm{C}{ }^{39}$. In our samples, taking also into account the results from XRD, the first peak at $813^{\circ} \mathrm{C}$ corresponds to a hexagonal to orthorhombic phase transition h-YMO $\rightarrow$ oYMO, whereas the second peak at $918{ }^{\circ} \mathrm{C}$ corresponds to the opposite, an orthorhombic to hexagonal phase transition o-YMO $\rightarrow \mathrm{h}$-YMO. Thereby, the amount of o-YMO phase detected in the sample YMO_800, which was annealed below the temperature of the first structural transition founded here $\left(813^{\circ} \mathrm{C}\right)$, has to be related with the longer time used for the sintering treatments in comparison with the relatively fast DSC experiments (without valleys). Regarding the loss of mass, it must be noted that the mass loss at the first transition at $813^{\circ} \mathrm{C}(\mathrm{h}->0)$ should be related with a variation in the oxygen content. However, the TG curve doesn't display 
any mass recovery neither above $813^{\circ} \mathrm{C}(\mathrm{h}->\mathrm{o})$ nor above at $918^{\circ} \mathrm{C}(\mathrm{o}->\mathrm{h})$. This feature ensures a similar oxygen content for samples obtained at different temperatures in spite these samples show different phase or different phase content. It is known the ability of the h-YMO structure to accommodate large concentrations of both oxygen vacancies and interstitial oxygen ions ${ }^{30}$, which determines the physical properties. Hence, this is a remarkable point to consider for the following characterization.

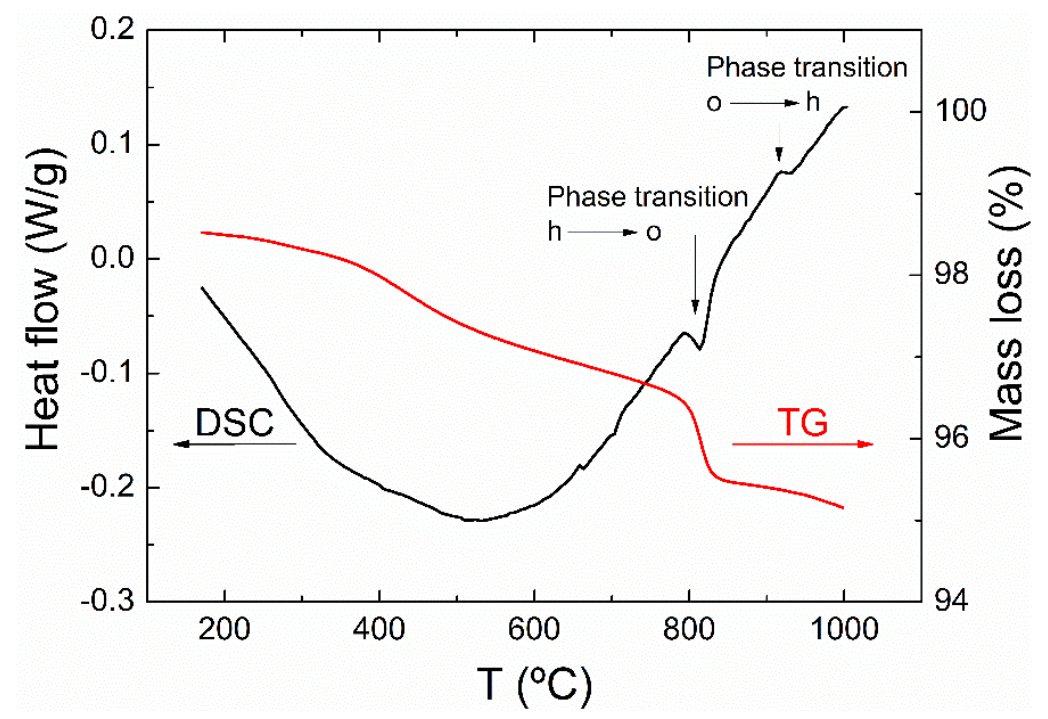

Figure 3. Differential Scanning Calorimetry (DSC) and Thermogravimetric (TG) curves of the as-milled nanostructured h-YMO powder.

\subsection{Dielectric measurements}

In order to study the processes related with the transport and the polarization effects, we discuss here the results of complex impedance measurements performed in the complete set of sintered samples. For this purpose, we will inspect the real parts of the dielectric permittivity $\left(\varepsilon^{\prime}\right)$ and the electrical conductivity $\left(\sigma^{\prime}\right)$, and additionally, the complex parts of the electric modulus $\left(\mathrm{M}^{\prime \prime}\right)$ and the impedance $\left(\mathrm{z}^{\prime \prime}\right)$ as a function of frequency. Figure 4 shows the frequency dependence of the real part of the dielectric permittivity (left panel) and the conductivity (right panel) at different temperatures for the family of samples. It can be observed that the dielectric permittivity $\left(\varepsilon^{\prime}\right)$ increases as the frequency decreases for all the samples, and the characteristic frequency where $\varepsilon^{\prime}$ upturns also rises as temperature increases, which points to the thermally activated behavior of this feature. The increase of $\varepsilon^{\prime}$ at the lowest frequencies is a characteristic mark of polarization effects at the electrode ${ }^{40}$. It is noteworthy that this process occurs at higher frequencies for the samples in which there is a larger content of the orthorhombic phase, i.e. the mixed samples YMO_800 and YMO_900, indicating a lower relaxation time in these cases.

In the $\sigma^{\prime}$ representation, which is displayed in right panel of figure 4 , it is meaningful to remark the large difference between $\sigma^{\prime}$ values of samples with different o-YMO phase content, in comparison with the samples with only $\mathrm{h}-\mathrm{YMO}$ phase. Hence, the two mixed samples with larger content of the o-YMO phase, YMO_800 and YMO_900 samples, display higher values (almost one order of magnitude for YMO_900 sample) than the samples with only h-YMO phase, YMO_1100 and YMO_1000 samples. Another feature can be observed by inspecting this representation: for YMO 1100 and YMO 1000 samples, $\sigma^{\prime}$ is almost constant for a broad frequency range and both samples show the "universal response" characterized by Jonscher's power law ${ }^{41}$ : 


$$
\sigma^{\prime}=\sigma_{\mathrm{dc}}+\sigma_{0} \omega^{\mathrm{n}}
$$

However, both YMO_800 and YMO_900 samples with high o-YMO phase content display a characteristic shoulder at intermediate frequencies, which could be related with a blockingrectifying effect at the electrode, although the universal response is also shown ${ }^{42}$.

Therefore, as it was concluded from the $\varepsilon^{\prime}$ analysis, clear differences can be easily noticed between samples with different o-YMO phase content. These features suggest that the changes are due to differences in the transport mechanism between the orthorhombic and hexagonal phases.

Up to this point, nothing has been mentioned about the sample sintered at the lowest temperature, YMO_700 sample, which displays features characteristic of both type of behaviors. The low frequency relaxation, which is characteristic of samples with o-YMO phase at intermediate frequencies, is also present, although it is significantly smoother. On the other hand, the values of $\varepsilon^{\prime}$ at high frequencies are closer to those shown by samples without orthorhombic phase, whereas $\sigma_{\mathrm{dc}}$ displays intermediate values between both type of samples. Therefore, in view of these features we must conclude that the YMO_700 sample contains a small fraction of o-YMO phase, which was already hinted by the X-ray diffraction measurements.
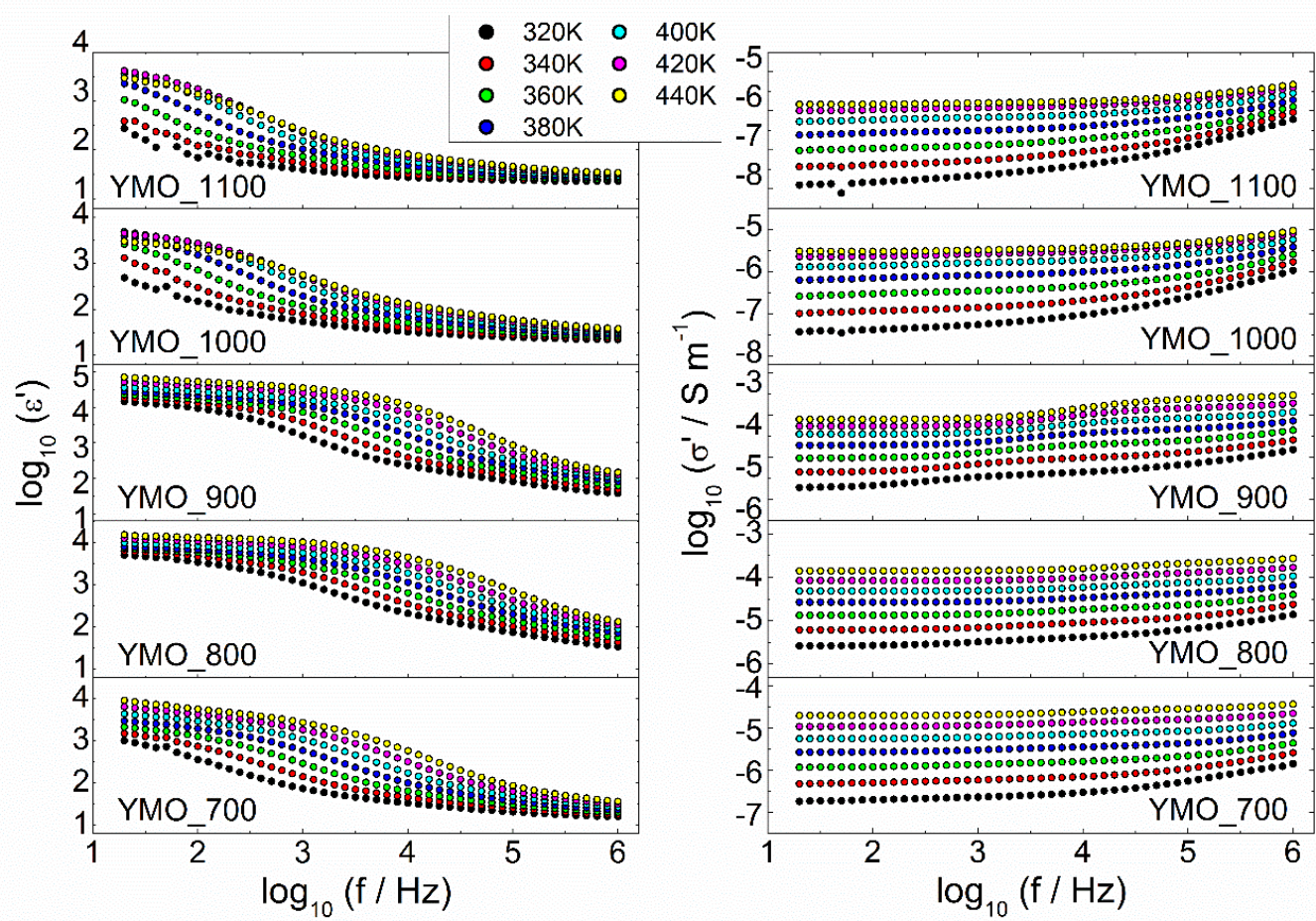

Figure 4. Temperature and frequency dependences of the real parts of dielectric permittivity $\varepsilon^{\prime}$ (left panel) and conductivity $\sigma^{\prime}$ (right panel) for all samples.

For a better understanding of the different electrical properties found in samples with dissimilar o-YMO phase content, we analyzed the imaginary parts of both the complex impedance ( $\left.z^{\prime \prime}\right)$ and the electrical modulus ( $\left.\mathrm{M}^{\prime \prime}\right)$. Figure 5 (left panel) displays the temperature and frequency dependences of the imaginary part of the impedance $\left(z^{\prime \prime}\right)$. In this representation, the two relaxation peaks at different frequencies can be more easily distinguished. For the samples with only h-YMO phase (YMO_1100 and YMO_1000), both relaxations are observed at high temperatures. Meanwhile, in the case of samples with larger o-YMO phase content, both relaxations appear at low temperatures. As we have already stated above, the low-frequency 
relaxation is related with an extrinsic Maxwell-Wagner type relaxation at the interface between the sample and the electrodes. This type of relaxation reflects the formation of Schottky-type barriers at the electrode-sample interface, which are consequence of differences between the work functions of the electrode material and the sample ${ }^{42}$. By inspecting the imaginary part of the electrical modulus $\left(\mathrm{M}^{\prime \prime}\right)$ in the right panel of figure 5, the trend displayed for the highfrequency relaxation is more evident, since this representation is less sensitive to the processes at low frequencies such as those related with the electrode interface. In this representation, it can be easily observed that the position of the high frequency peak correlates well with the proportion of h-YMO to o-YMO phases of each sample. Thereby, YMO_1000 and YMO_1100 samples show the maximum of the characteristic peak around $3 \mathrm{kHz}$ at $320 \mathrm{~K}$, whereas YMO_800 and YMO_900 samples show this maximum above $1 \mathrm{MHz}$ at the same temperature. Finally, the sample with a small fraction of o-YMO (YMO_700 sample) displays this characteristic maximum above $30 \mathrm{kHz}$, approximately.

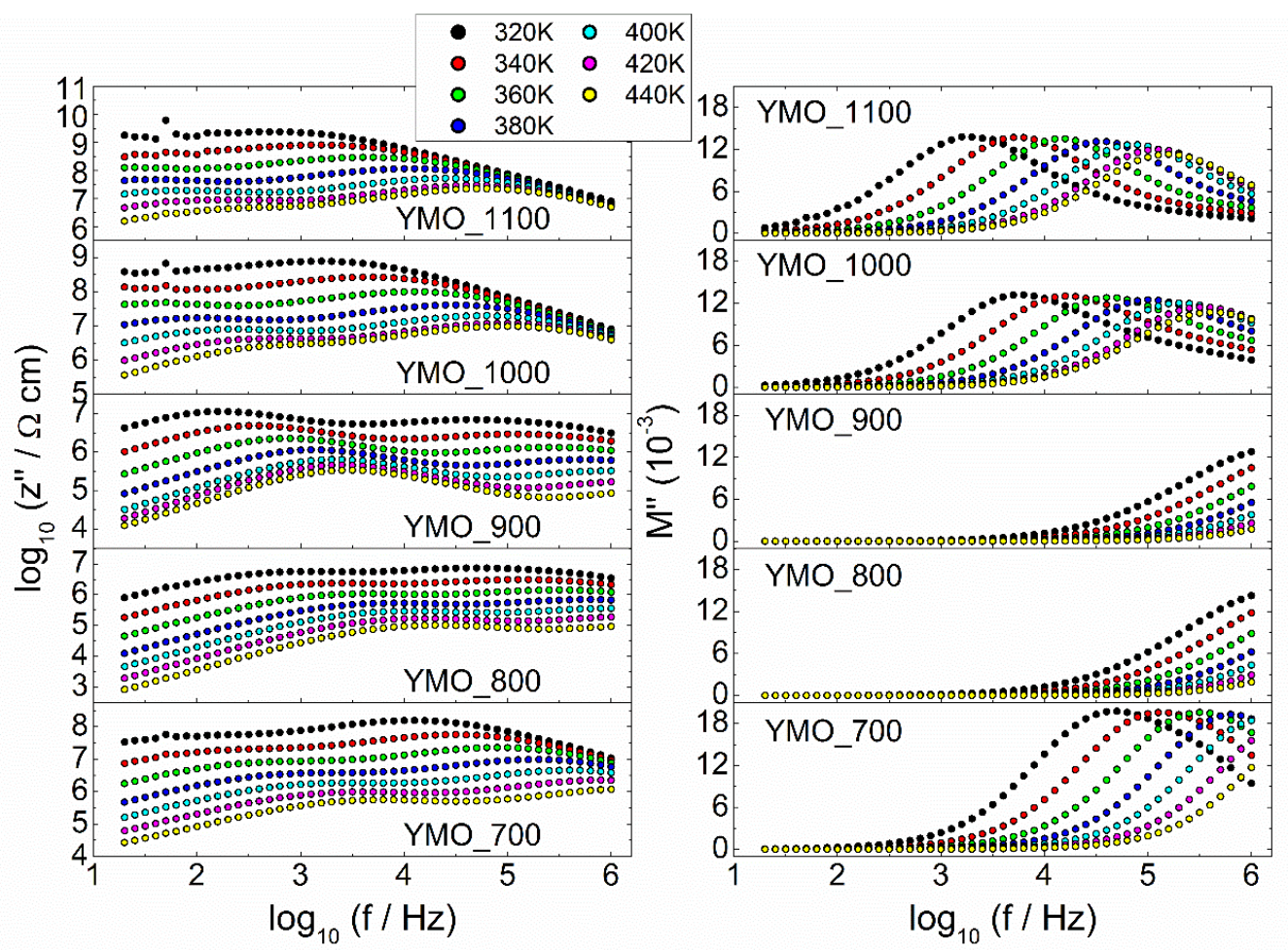

Figure 5. Temperature and frequency dependences of the imaginary parts of the impedance $z^{\prime \prime}$ (left panel) and electrical modulus $M^{\prime \prime}$ (right panel) for all samples.

In figure 6 we compare the real parts of dielectric permittivity $\left(\varepsilon^{\prime}\right)$ and conductivity $\left(\sigma^{\prime}\right)$ at two different temperatures ( $340 \mathrm{~K}$ and $440 \mathrm{~K}$ ) for the samples studied in this work. In the conductivity plot (right), we can distinguish two block regions, which are defined as the regions with a slope change in the curve. Also, the values of conductivity increase with the temperature, which agrees well with the curves of the imaginary part of impedance. On the other hand, there is a region of constant conductivity at low frequencies which is a characteristic feature of the electronic transport. Also, the enhanced conductivity in samples with coexistence of both phases confirms that the orthorhombic phase is better conductor than the hexagonal one. Since, the electrical conductivity in $\mathrm{YMnO}_{3}$, for both hexagonal and orthorhombic structure, has been assigned to $\mathrm{p}$-type hopping between non stoichiometric $\mathrm{Mn}$ cations $\left(\mathrm{Mn}^{3+} \text { and } \mathrm{Mn}^{4}\right)^{21,43}$. The higher conductivity values associated with o-YMO would indicate either a higher content of $\mathrm{Mn}^{4+}$ or a higher mobility of holes associated to $\mathrm{Mn}^{4+}$ ions. 

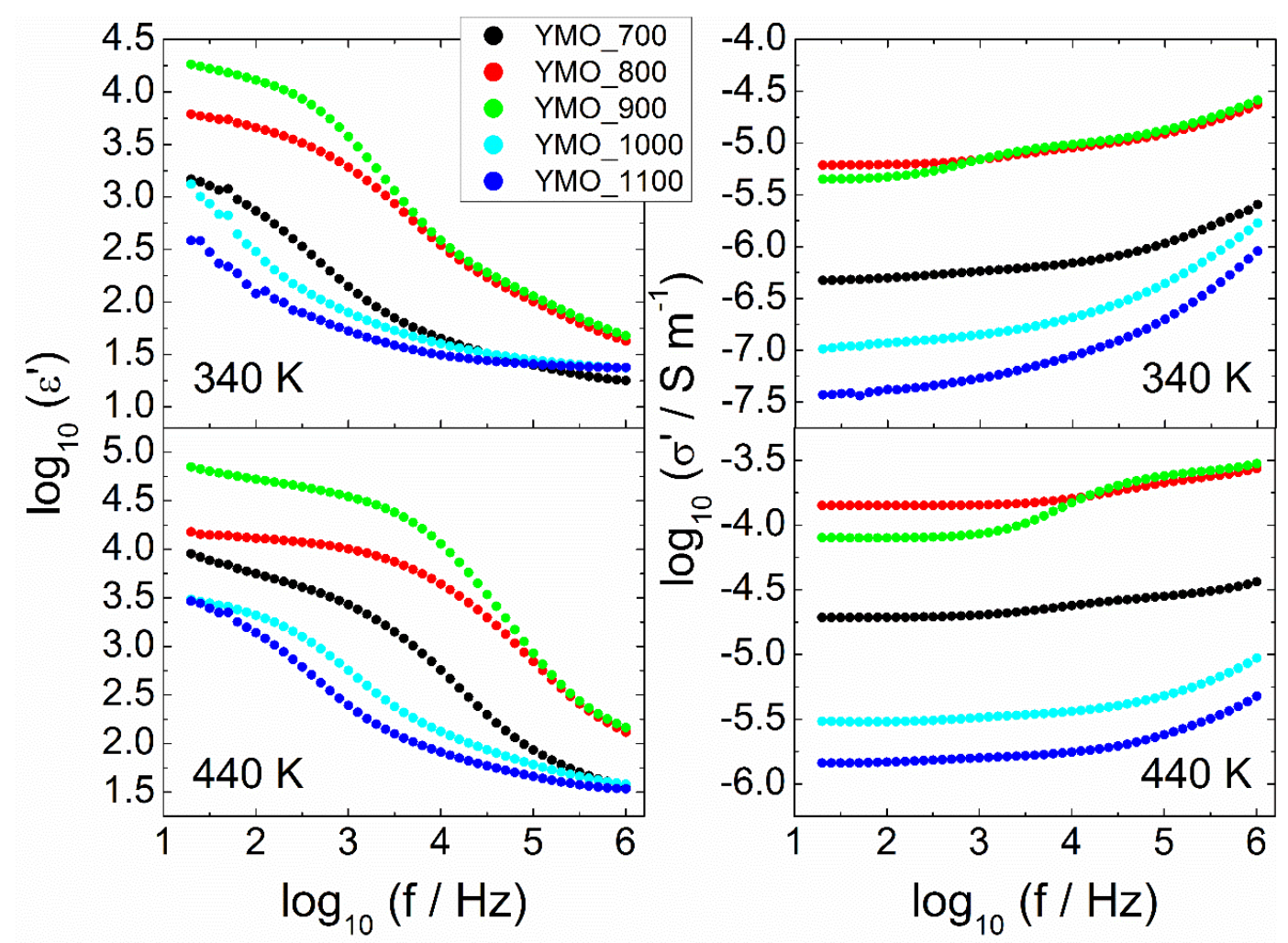

Figure 6. Real parts of permittivity $\varepsilon^{\prime}$ (left panel) and conductivity $\sigma^{\prime}$ (right panel) as a function of frequency for all samples at $340 \mathrm{~K}$ and $440 \mathrm{~K}$.

The activation energies associated to the intrinsic conductivity process are displayed in figure 7 (left panel). The conductivity values were taken at enough high frequencies to reflect the transport without the electrode influence and additionally, these were compared with values obtained from fitting curves (solid lines in right panel of figure 7) when it was possible. The activation energy values $E_{a}$ obtained from the Arrhenius plots for all samples are similar to those previously reported ${ }^{20,43-45}$ and they show a clear trend: while the $\mathrm{E}_{\mathrm{a}}$ are $0.49 \mathrm{eV}$ and $0.48 \mathrm{eV}$ for samples with only $h-Y M O$ phase, the value of the $E_{a}$ is slightly lower for the mixed samples which also contain o-YMO phase, about 0.42-0.45 eV. Similar results were also calculated from the electrical modulus $\mathrm{M}^{\prime \prime}$ curves. The complex impedance plots shown in the figure 7 (right panel) display the two characteristic semicircles: the left one corresponding to high frequencies, which is associated to the transport through $\mathrm{YMnO}_{3}$, while the right one corresponds to a rectifying effect at the electrode. As a result of the higher conductivity values displayed by mixed samples, YMO_800 and YMO_900, the semicircles corresponding to the bulk conductivity are comparable or smaller than those corresponding to the rectifying effect at the electrode. These data were fitted to an equivalent circuit of two RC elements combined in series (see inset of right panel). In both RC elements, the non-ideal behavior was accounted by including a CPE (Constant-Phase element) in parallel with the ideal capacitor for the bulk and instead of the ideal capacitor for the electrode ${ }^{42}$. The parameters obtained from these fits are summarized in the table 1. 

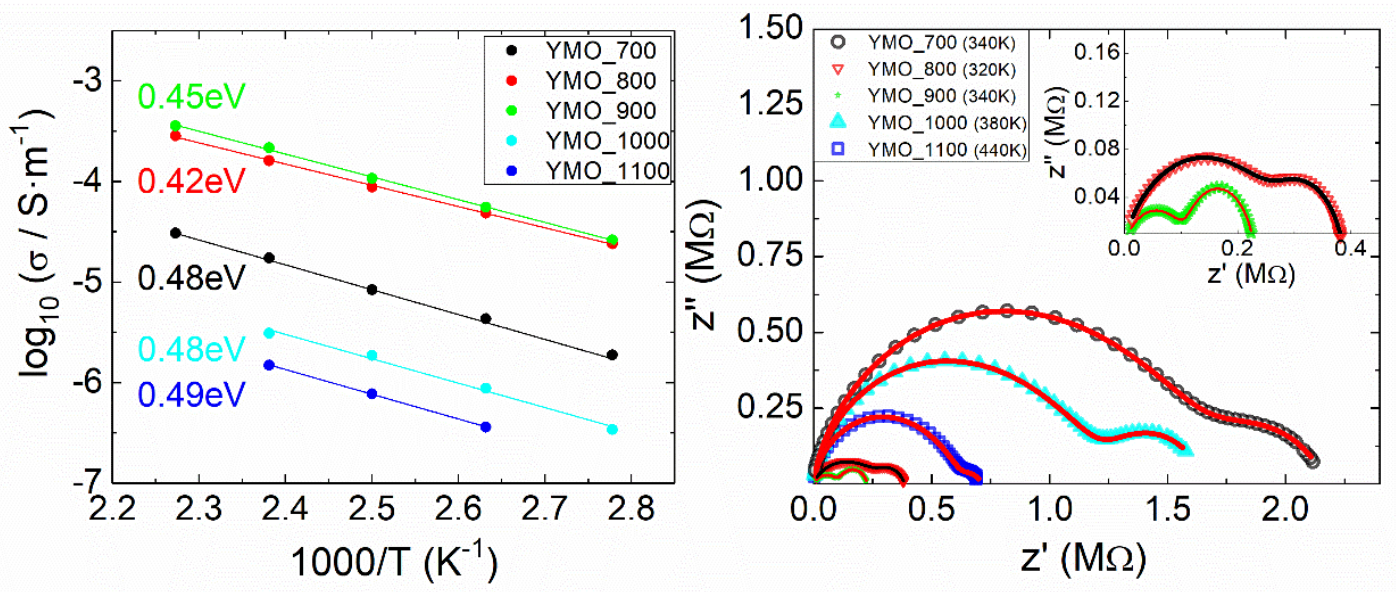

Figure 7. Activation energy values (left panel) and complex impedance plots (right panel) for all samples. Solid lines represent the Arrhenius plots (left panel) and the fittings to the equivalent circuit included in the right panel (see text and table 1 for details).

\begin{tabular}{cccccc}
\hline Parameter & YMo_700 (340 K) & YMO_800 (320 K) & YMO_900 (340 K) & YMO_1000 (340 K) & YMO_1100 (440 K) \\
\hline \hline$R_{1}(\Omega)$ & $1.71 \times 10^{6}$ & $2.8 \times 10^{5}$ & $1.1 \times 10^{5}$ & $1.2 \times 10^{6}$ & $6.5 \times 10^{5}$ \\
\hline$Q_{1}\left(\Omega^{-1} \cdot \mathrm{s}^{n}\right)$ & $6.8 \times 10^{-10}$ & $3.0 \times 10^{-9}$ & $3.5 \times 10^{-9}$ & $8.7 \times 10^{-10}$ & $9.0 \times 10^{-10}$ \\
\hline$n_{1}$ & 0.52 & 0.55 & 0.58 & 0.53 & 0.55 \\
\hline$C_{1}\left(\Omega^{-1} \cdot s\right)$ & $1.31 \times 10^{-12}$ & $1.0 \times 10^{-12}$ & $8.1 \times 10^{-13}$ & $2.3 \times 10^{-12}$ & $2.5 \times 10^{-12}$ \\
\hline$R_{2}(\Omega)$ & $3.2 \times 10^{5}$ & $1.02 \times 10^{5}$ & $1.2 \times 10^{5}$ & $7.1 \times 10^{5}$ & $5.8 \times 10^{4}$ \\
\hline$Q_{2}\left(\Omega^{-1} \cdot s^{n}\right)$ & $9.8 \times 10^{-9}$ & $1.23 \times 10^{-8}$ & $1.4 \times 10^{-8}$ & $1.1 \times 10^{-7}$ & $7.5 \times 10^{-8}$ \\
\hline$n_{2}$ & 0.82 & 0.81 & 0.82 & 0.50 & 0.69 \\
\hline$\chi^{2}$ & 0.00023 & 0.00013 & 0.0001 & 0.00016 & 0.00018 \\
\hline
\end{tabular}

Table 1 Parameters obtained by fitting the experimental data to the equivalent circuit described in the text with the Zview ${ }^{\circledR}$ software. The impedance of CPE is given by $Z_{C P E}=Q^{-1} \cdot(j \omega)^{-n}$

\subsection{Magnetic characterization}

Figure 8 (left panel) displays the ZFC and FC magnetization curves of all the samples obtained under an applied magnetic field of $\mathrm{H}=100$ Oe. Additionally, the right panel of figure 8 shows the temperature dependence of the inverse of the magnetic susceptibility $(\mathrm{X})$, allowing the identification of transition temperature by means of the slope change of the curve ${ }^{46-49}$. The transition temperatures of each phase are shown in table 2. As an example, the second derivative of $1 / \chi$ curves of samples YMO_800 and YMO_1100 are shown in the corresponding insets. It can be observed that in samples with just hexagonal phase, there is only one slope change in the curve, leading to a minimum in the second derivative. These minima give transition temperatures of 67.9 and $68.8 \mathrm{~K}$ for YMO 1000 and YMO 1100 , respectively, which are close to the Néel temperature associated to the hexagonal phase $\left(T_{N}{ }^{h}\right)^{5,13,15,18,22,47,50}$. On the other hand, the mixed samples present two slope changes, leading to a maximum and a minimum in the second derivative curve. The minimum values, which are 70.9, 73.4 and $76 \mathrm{~K}$ for YMO_700, YMO_800 and YMO_900, respectively, are close to $T_{N}{ }^{h}{ }^{18,51}$, while the maximum values of the curve, which are $37.8,40.7$ and $41.9 \mathrm{~K}$, are close to the Néel temperature associated to the orthorhombic phase of $\mathrm{YMO}\left(\mathrm{T}_{\mathrm{N}}{ }^{\circ}\right)^{24,52,53}$. These features suggest a slight effect of the sintering temperature on the increase of the transition temperature for both mixed and single-phase samples. Nevertheless, it seems that there is an additional effect that enhances the hexagonal transition temperature in the mixed samples, and it drops off for single-phase samples YMO_1000 and YMO_1100. In order to measure indirectly the strength of the antiferromagnetic 
interaction ${ }^{13}$, we have obtained the Curie-Weiss temperatures, $\theta_{p}$, (shown in Table 2 ) by means of the linear fitting of the $1 / \chi$ curve in the paramagnetic region. These fits are shown in figure 8 (right panel) as linear fits from $200 \mathrm{~K}$ to $300 \mathrm{~K}$. Noteworthily, the obtained values of $\theta_{\mathrm{p}}$ for both YMO_1000 and YMO_1100 samples are fairly higher than those corresponding to the mixed samples. All these values are in agreement with the values previously reported, being lower for the orthorhombic phase than for the hexagonal phase 13,15,23,24,50,51,54. Thereby, with the enhancement of the hexagonal transition temperature, the Curie-Weiss temperatures for mixed samples show smaller AF interaction. However, the sample YMO_700, that contains only traces of o-YMO, presents a decreased Curie-Weiss temperature while the transition temperature increases in respect to single-phase samples. We suggest that the presence of the orthorhombic phase could be the reason for this behavior, giving place to an interaction between both o-YMO and $\mathrm{h}-\mathrm{YMO}$ phases that changes the AF interaction strength and shifts the transition temperature to higher temperatures.
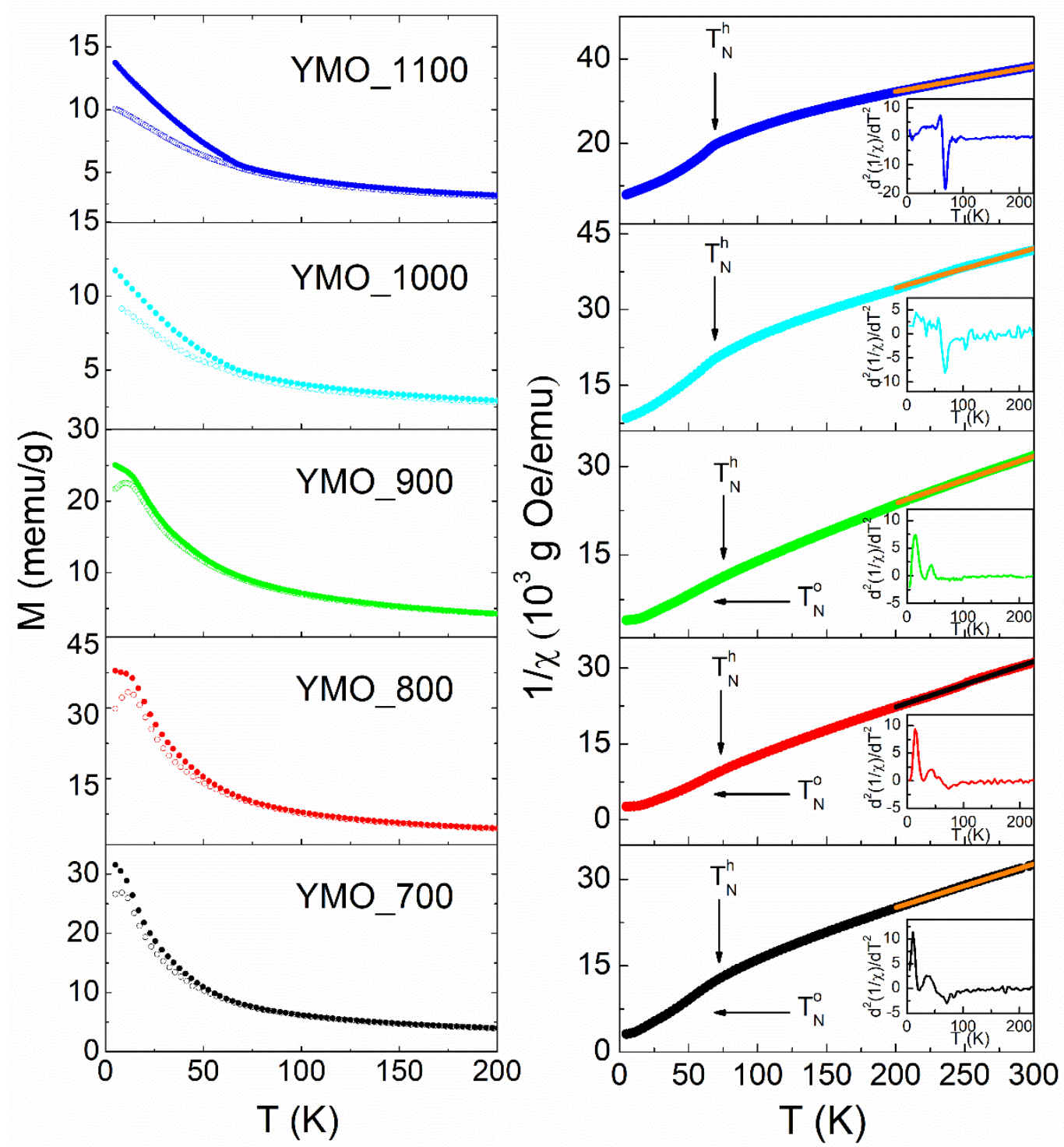

Figure 8. Zero-field cooled (solid symbols) and field cooled (open symbols) curves under an external magnetic field $\mathrm{H}=$ 100 Oe (left panel). The inverse of susceptibility $\chi$ (right panel) with the Curie -Weiss linear fits (solid lines) for all samples. The insets in the right panel correspond to the second derivative of $1 / \chi$. 


\begin{tabular}{cccc}
\hline Sample & $\begin{array}{c}\text { Néel temperature (K) } \\
h-Y M O T_{N}{ }^{h}\end{array}$ & $\begin{array}{c}\text { Néel temperature (K) } \\
\text { o-YMO } T_{N}{ }^{\circ}\end{array}$ & $\begin{array}{c}\text { Curie-Weiss temperature } \\
\Theta_{P}(K)\end{array}$ \\
\hline \hline YMO_700 & $70.9 \pm 0.2$ & $37.8 \pm 0.2$ & $-131.4 \pm 0.2$ \\
\hline YMO_800 & $73.4 \pm 0.2$ & $40.7 \pm 0.2$ & $-47.7 \pm 0.2$ \\
\hline YMO_900 & $76 \pm 0.5$ & $41.9 \pm 0.2$ & $-97.9 \pm 0.2$ \\
\hline YMO_1000 & $67.9 \pm 0.2$ & - & $-239.3 \pm 0.2$ \\
\hline YMO_1100 & $68.8 \pm 0.2$ & - & $-344.6 \pm 0.2$ \\
\hline
\end{tabular}

Table 2. Néel temperatures for each phase and Curie-Weiss temperatures for all samples.

Meanwhile, comparing the magnetization curves at low temperature we observe that $\mathrm{YMO} 800$ and YMO 900 samples present larger values of magnetization than the corresponding to samples with only h-YMO phase (in agreement with the observed values in the hysteresis loops, which are further discussed). This feature agrees well with previous experimental results where the $\mathrm{h}$-YMO shows lower magnetization values than o-YMO. These different values reflect the different antiferromagnetic arrangement, being type-E for the orthorhombic one and type-A for the hexagonal structure 23,24. Another singular feature related with the different antiferromagnetic order is the spin-glass-like behavior observed for all the samples that include O-YMO phase: strong irreversibility between ZFC and FC curves as well as a peak at low temperature in the ZFC magnetization curve. The maximum at $11 \mathrm{~K}$ would correspond to the freezing temperature ${ }^{26}$. This spin-glass-like behavior has been associated to an intrinsic disorder induced by the presence of $\mathrm{Mn}^{4+}$ ions related with the stabilization of the orthorhombic phase 24.

Finally, it should be noted that the $1 / \chi$ curves differs from those of a pure antiferromagnetic material ${ }^{46}$, since the slope change below the transition temperature suggests a ferromagnetic contribution. In particular, YMO_700, YMO_1000 and YMO_1100 samples show a sharper slope change of the curve than the observed for YMO 800 and YMO 900 . It has been recently reported FM contribution for both o-YMO and h-YMO phase, although its origin is not clear. Different authors have suggested that it could be due to a non-stoichiometry of oxygen, disorder allocated at grain boundaries, or an alternative explanation could be the double-exchange interaction, which is a characteristic mechanism in perovskite oxides 4-7,23,26,52,55. This ferromagnetic contribution observed in $1 / x$ curves should be also evident in the hysteresis loops. Thus, to complete the magnetic characterization, hysteresis loops at low temperature (5 K) were obtained for all samples (after field-cooling from room temperature under an applied magnetic field of $10 \mathrm{kOe}$ ). Figure 9 shows the hysteresis loops of two significant samples studied in this work: the sample with only hexagonal phase sintered at maximum temperature (YMO_1100) and the sample with the maximum o-YMO content (YMO_900). Both samples display a small hysteretic behavior, indicating the presence of the ferromagnetic contribution. Additionally, both loops present a certain shift in the field axis as it is shown in the insets. This is a characteristic feature of the exchange bias phenomena, which arises when two materials with significantly different magnetic anisotropy, typically a ferromagnet and an antiferromagnet, are exchanged coupled at their mutual interface ${ }^{56,57}$. Hence, the existence of exchange bias also evidences the presence of a ferromagnetic contribution interacting with the antiferromagnetic phase. 

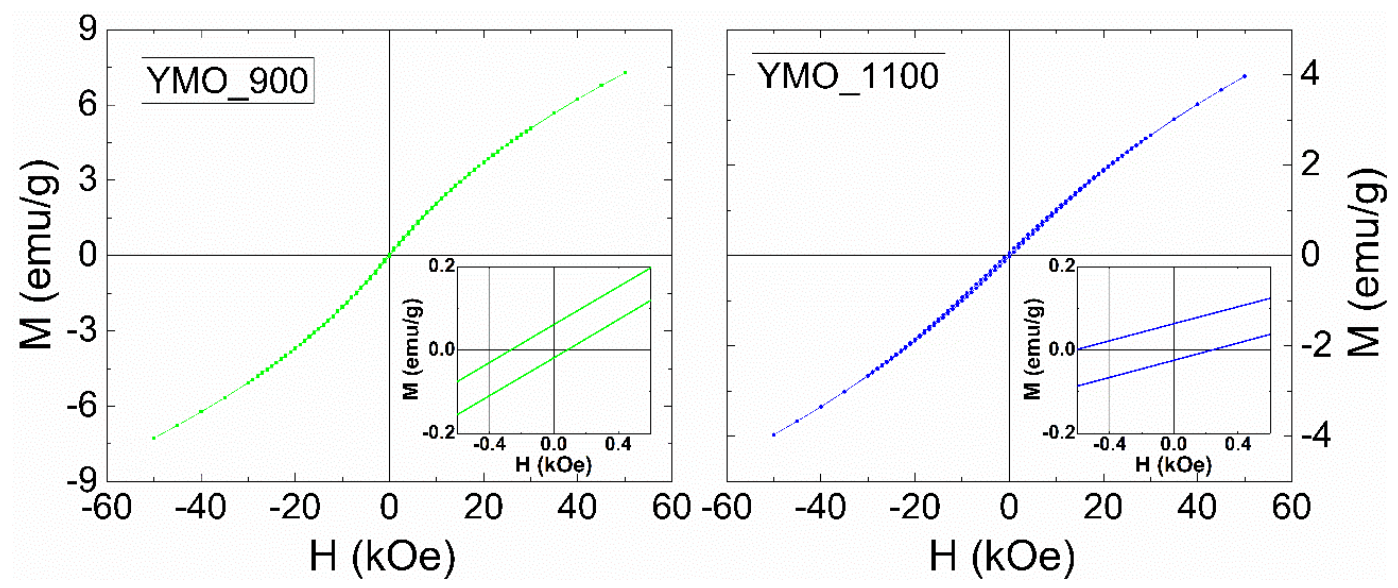

Figure 9. Hysteresis loops for the samples YMO_900 (left panel) and YMO_1100 (right panel) at 5 K. The insets in both panels include the zoom at the origin of the axes.

The results presented in this section are valuable in view of the interest displayed by the multiferroic $\mathrm{YMnO}_{3}$. As we have already commented, there are several studies where the $\mathrm{p}$-type conductivity or magnetodielectric properties are analyzed offering inconsistent results. However, up to now no experimental or theoretical works have considered thoroughly the effect of a metastable orthorhombic phase on the physical properties of $\mathrm{YMnO}_{3}$. Considering that the metastable phase has been tuned easily by selecting a proper thermal treatment and the large effects it has on its properties, our results become very relevant due to the large interest of the potential applications of $\mathrm{YMnO}_{3}$.

\section{Conclusions}

In summary, we have obtained a family of $\mathrm{YMnO}_{3}$ perovskites where the amount of the metastable orthorhombic phase is controlled by means of different sintering steps. Measurements of the magnetic susceptibility and dielectric spectroscopy have been performed to investigate the role played by the orthorhombic $\mathrm{YMnO}_{3}$ phase in polycrystalline samples on its dielectric and magnetic properties. The magnetic characterization for both o-YMO and h-YMO structures displays a ferromagnetic contribution evidenced by the observation of exchange bias in the hysteresis loops. A clear trend is observed through the impedance spectroscopy characterization, where the samples with higher o-YMO content display higher conductivity. The larger values of electrical conductivity as well as the spin-glass-like behavior of samples containing orthorhombic $\mathrm{YMnO}_{3}$ can be related to the presence of $\mathrm{Mn}^{4+}$. These results demonstrate that by controlling the thermal treatment, and therefore the content of the metastable phase, the physical properties of multiferroic $\mathrm{YMnO}_{3}$ can be tuned.

\section{Acknowledgements}

This work was supported by the Spanish Ministry of Economy and Competitiveness (grant 201458034-R). M. Sanchez-Perez thanks to the Spanish Ministry of Economy and Competitiveness for her predoctoral fellowship.

\section{References}

1 M. Počuča-Nešić, Z. Marinković Stanojević, Z. Branković, P. Cotič, S. Bernik, M. Sousa Góes, B. A. Marinković, J. A. Varela, and G. Branković, Journal of Alloys and Compounds 552, 451 (2013).

2 T. Ahmad, I. H. Lone, and M. Ubaidullah, RSC Advances 5, 58065 (2015). 
M. Fiebig, T. Lottermoser, D. Meier, and M. Trassin, Nature Reviews Materials 1, 16046 (2016).

Z. Branković, G. Branković, M. Počuča-Nešić, Z. Marinković Stanojević, M. Žunić, D. Luković Golić, R. Tararam, M. Cilense, M. A. Zaghete, Z. Jagličić, M. Jagodič, and J. A. Varela, Ceramics International 41, 14293 (2015).

K. Bergum, H. Okamoto, H. Fjellvåg, T. Grande, M.-A. Einarsrud, and S. Selbach, Dalton Transactions 40, 7583 (2011).

X. Marti, V. Skumryev, A. Cattoni, R. Bertacco, V. Laukhin, C. Ferrater, M. V. GarcíaCuenca, M. Varela, F. Sánchez, and J. Fontcuberta, Journal of Magnetism and Magnetic Materials 321, 1719 (2009).

S. Cheng, M. Li, S. Deng, S. Bao, P. Tang, W. Duan, J. Ma, C. Nan, and J. Zhu, Advanced Functional Materials 26, 3589 (2016).

A. S. Gibbs, K. S. Knight, and P. Lightfoot, Physical Review B 83, 094111 (2011).

D. Khomskii, Physics 2 (2009).

B. B. Van Aken, T. T. M. Palstra, A. Filippetti, and N. A. Spaldin, Nature Materials 3, 164 (2004).

A. Bogusz, A. D. Müller, D. Blaschke, I. Skorupa, D. Bürger, A. Scholz, O. G. Schmidt, and H. Schmidt, AIP Advances 4, 107135 (2014).

T. Choi, Y. Horibe, H. T. Yi, Y. J. Choi, W. Wu, and S. W. Cheong, Nature Materials 9, 253 (2010).

S. Chauhan, A. K. Singh, S. K. Srivastava, and R. Chandra, Journal of Magnetism and Magnetic Materials 414, 187 (2016).

M. Muneeswaran, J. W. Jang, B. C. Choi, J. H. Jeong, and N. V. Giridharan, Journal of Materials Science: Materials in Electronics 28, 16788 (2017).

L. Jeuvrey, O. Peña, A. Moure, and C. Moure, Journal of Magnetism and Magnetic Materials 324, 717 (2012).

P. Ren, H. Fan, and X. Wang, Applied Physics Letters 103, 152905 (2013).

H. Pang, F. Zhang, M. Zeng, X. Gao, M. Qin, X. Lu, J. Gao, J. Dai, and Q. Li, Npj Quantum Materials 1, 16015 (2016).

T.-C. Han, W.-L. Hsu, and W.-D. Lee, Nanoscale Research Letters 6, 201 (2011).

E. Dagotto, T. Hotta, and A. Moreo, Physics Reports 344, 1 (2001).

M. Tomczyk, P. M. Vilarinho, A. Moreira, and A. Almeida, Journal of Applied Physics 110, 064116 (2011).

M. C. Sekhar and N. V. Prasad, Ferroelectrics 345, 45 (2006).

T. Katsufuji, S. Mori, M. Masaki, Y. Moritomo, N. Yamamoto, and H. Takagi, Physical Review B 64, 104419 (2001).

A. Muñoz, J. Alonso, M. J. Martínez-Lope, M. T. Casáis, J. Martínez, and M. T. FernándezDíaz, Physical Review B 62 (2000).

A. Muñoz, J. A. Alonso, M. T. Casais, M. J. Martínez-Lope, J. L. Martínez, and M. T. Fernández-Díaz, Journal of Physics: Condensed Matter 14, 3285 (2002).

W.-C. Yi, S.-I. Kwun, and J.-G. Yoon, Journal of the Physical Society of Japan 69, 2706 (2000).

I. Iliescu, M. Boudard, L. Rapenne, O. Chaix-Pluchery, and H. Roussel, Applied Surface Science 306, 27 (2014).

I. lliescu, M. Boudard, O. Chaix-Pluchery, L. Rapenne, and H. Roussel, Journal of Solid State Chemistry 220, 245 (2014).

B. Lorenz, Y. Q. Wang, Y. Sun, and C. W. Chu, Physical Review B 70, 212412 (2004).

S. H. Skjærvø, E. T. Wefring, S. K. Nesdal, N. H. Gaukås, G. H. Olsen, J. Glaum, T. Tybell, and S. M. Selbach, Nature Communications 7 (2016).

S. Skjærvø, D. Småbråten, N. Spaldin, T. Tybell, and S. Selbach, Physical Review B 98 (2018). 
E. Ruff, S. Krohns, M. Lilienblum, D. Meier, M. Fiebig, P. Lunkenheimer, and A. Loidl, Physical Review Letters 118, 036803 (2017).

X. Wang, D. Yang, H.-M. Zhang, C. Song, J. Wang, G. Tan, R. Zheng, S. Dong, S.-W. Cheong, and J. Zhang, Physical Review B 99, 054106 (2019).

N. Kumar Swamy, P. Kumar, M. Gupta, S. S. Samatham, V. Ganesan, V. Malik, and B. Das, Journal of Thermal Analysis and Calorimetry 119, 1191 (2015).

A. Durán, G. Guzmán, C. I. Ochoa-Guerrero, C. Herbert, R. Escudero, F. Morales, and R. Escamilla, Journal of Applied Physics 125, 034102 (2019).

Y. Zhao, Y. Rao, B. Luo, C. Chen, H. Xing, L. Niu, J. Wang, and K. Jin, The Journal of Physical Chemistry C 120, 22318 (2016).

S. Krohns and P. Lunkenheimer, Physical Sciences Reviews 4 (2019).

R. Thakur, R. Thakur, B. Arunachalam, V. Ganesan, and N. Gaur, Modern Physics Letters B 26, 1250201 (2012).

D. Nečas and P. Klapetek, Central European Journal of Physics 10 (2011).

B. Fu, W. Huebner, M. F. Trubelja, and V. S. Stubican, Journal of Materials Research 9, 2645 (2011).

R. Schmidt, in Ceramic Materials Research Trends (Nova Science Publishers, 2007), p. 325.

A. K. Jonscher, Journal of Physics D: Applied Physics 32, R57 (1999).

R. Schmidt, J. Ventura, E. Langenberg, N. Nemes, C. Munuera, M. Varela, M. GarciaHernandez, C. Leon, and J. Santamaria, Physical Review B 86, 035113 (2012).

U. Adem, N. Mufti, A. A. Nugroho, G. Catalan, B. Noheda, and T. T. M. Palstra, Journal of Alloys and Compounds 638, 228 (2015).

Y. Ma, Y. J. Wu, X. Ming Chen, J. Peng Cheng, and Y. Qi Lin, Ceramics International 35, 3051 (2009).

F. Wan, X. Lin, X. Bai, X. Han, K. Song, J. Zheng, and C. Cao, Journal of Materials Science: Materials in Electronics 27, 3082 (2016).

J. M. D. Coey, Magnetism and Magnetic Materials (Cambridge University Press, Cambridge, 2010).

A. A. Nugroho, N. Bellido, U. Adem, G. Nénert, C. Simon, M. O. Tjia, M. Mostovoy, and T. T. M. Palstra, Physical Review B 75, 174435 (2007).

L. P. Yang, A. M. Zhang, Y. Dai, and X. S. Wu, Journal of Materials Science: Materials in Electronics 28, 8872 (2017).

Y. Ma, Y. Chen, Z. Wang, X. Wang, and H. Liu, Journal of Materials Science: Materials in Electronics 29, 7287 (2018).

C. Zhang, J. Su, X. Wang, F. Huang, J. Zhang, Y. Liu, L. Zhang, K. Min, Z. Wang, X. Lu, F. Yan, and J. Zhu, Journal of Alloys and Compounds 509, 7738 (2011).

Z. J. Huang, Y. Cao, Y. Y. Sun, Y. Y. Xue, and C. W. Chu, Physical Review B 56, 2623 (1997).

Z. Chen, C.-L. Ma, F.-X. Wu, Y. B. Chen, J. Zhou, G.-L. Yuan, Z.-B. Gu, S.-T. Zhang, and Y.-

F. Chen, Applied surface science 257, 8033 (2011).

M. Iliev, B. Lorenz, A. Litvinchuk, Y. Q. Wang, Y. Sun, and C. W. Chu, Journal of Physics Condensed Matter 17, 3333 (2005).

R. Dhinesh Kumar and R. Jayavel, Materials Letters 113, 210 (2013).

N. Kumar, A. Gaur, and G. D. Varma, Journal of Alloys and Compounds 509, 1060 (2011). J. Nogués, J. Sort, V. Langlais, V. Skumryev, S. Suriñach, J. S. Muñoz, and M. D. Baró, Physics Reports 422, 65 (2005).

J. González, J. Andrés, R. Lopez Anton, J. De Toro, P. Normile, P. Muñiz, J. Manuel Riveiro, and J. Nogues, Chemistry of Materials 29, 5200 (2017). 

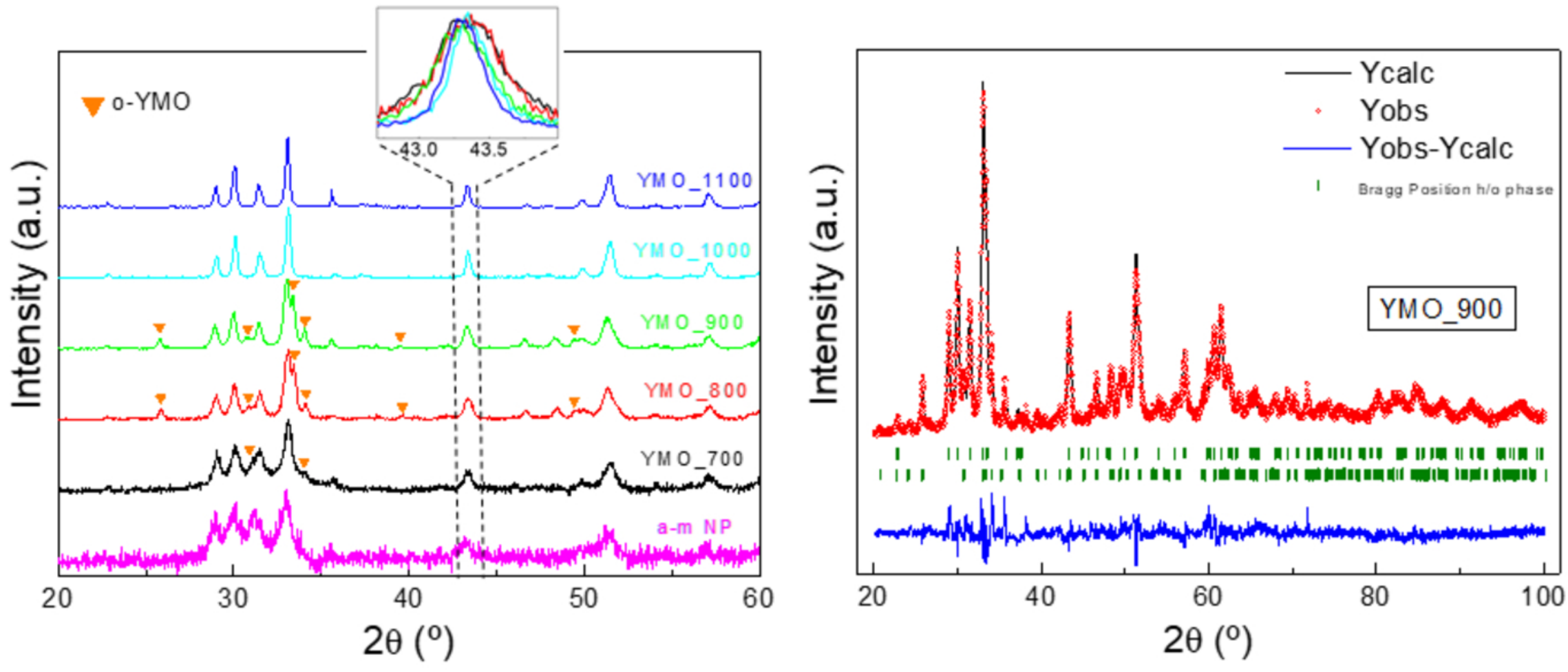

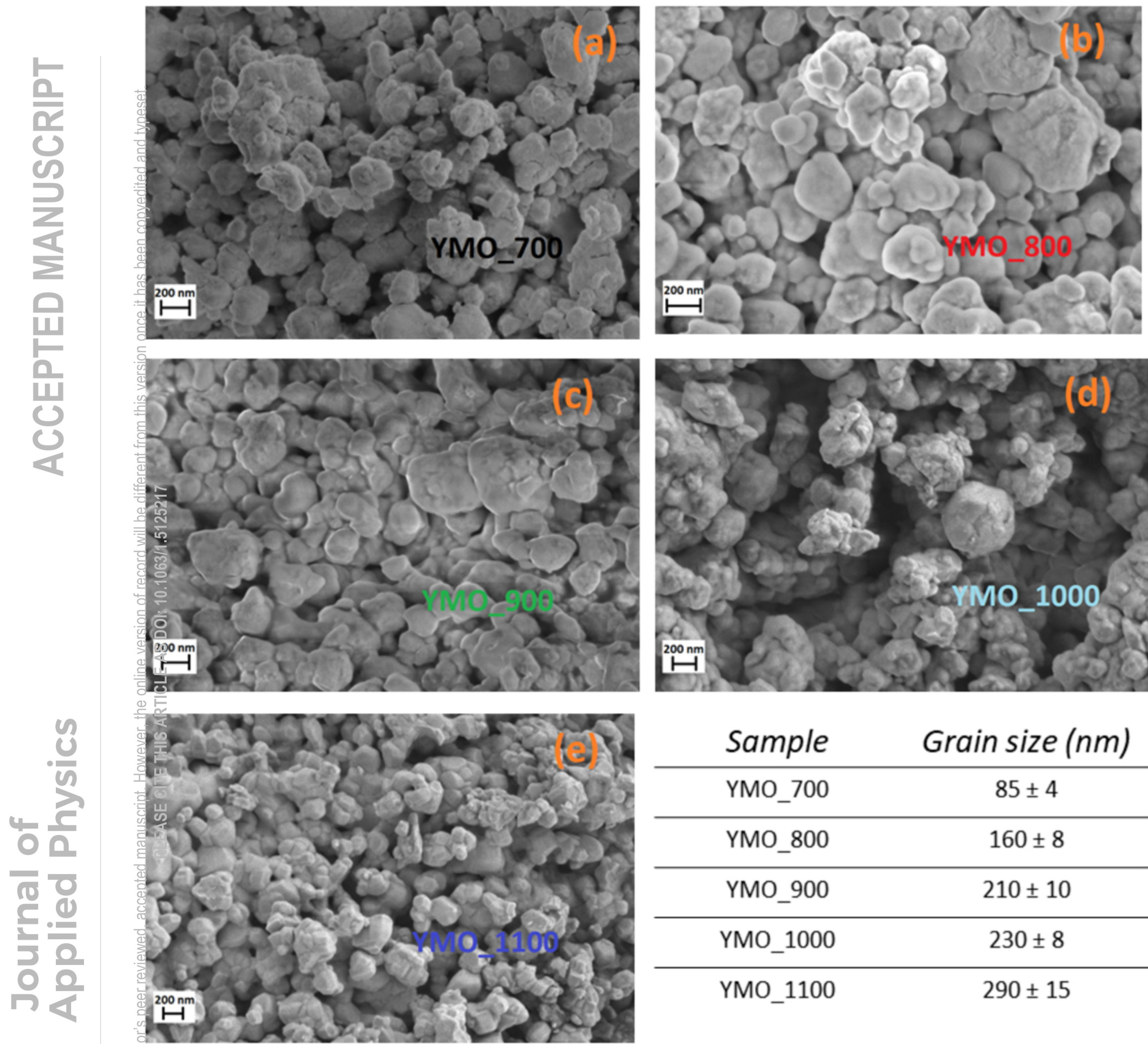

\begin{tabular}{cc} 
Sample & Grain size $(\mathrm{nm})$ \\
\hline YMO_700 & $85 \pm 4$ \\
\hline YMO_800 & $160 \pm 8$ \\
\hline YMO_900 & $210 \pm 10$ \\
\hline YMO_1000 & $230 \pm 8$ \\
\hline YMO_1100 & $290 \pm 15$
\end{tabular}

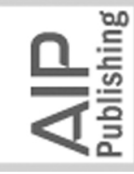




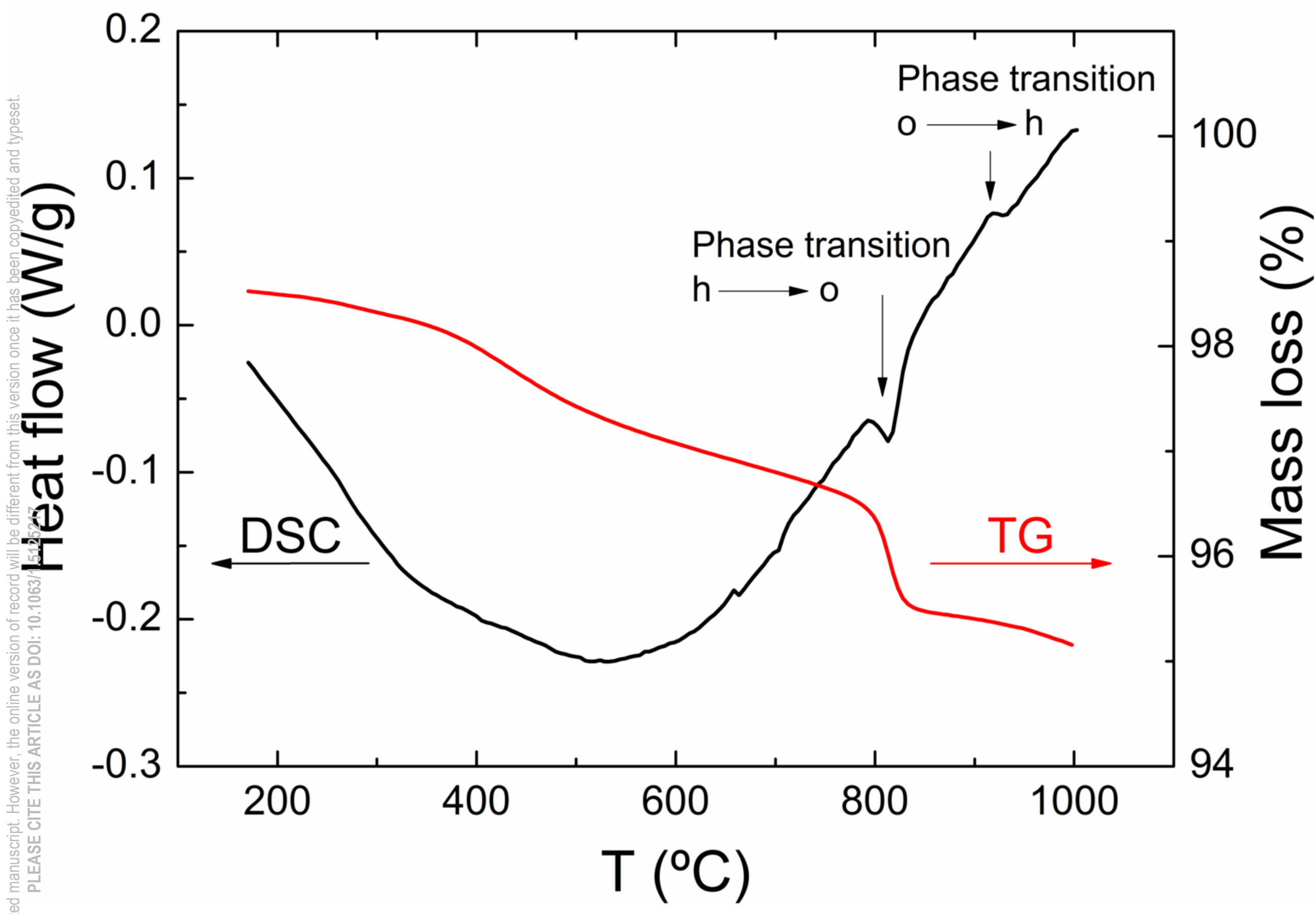




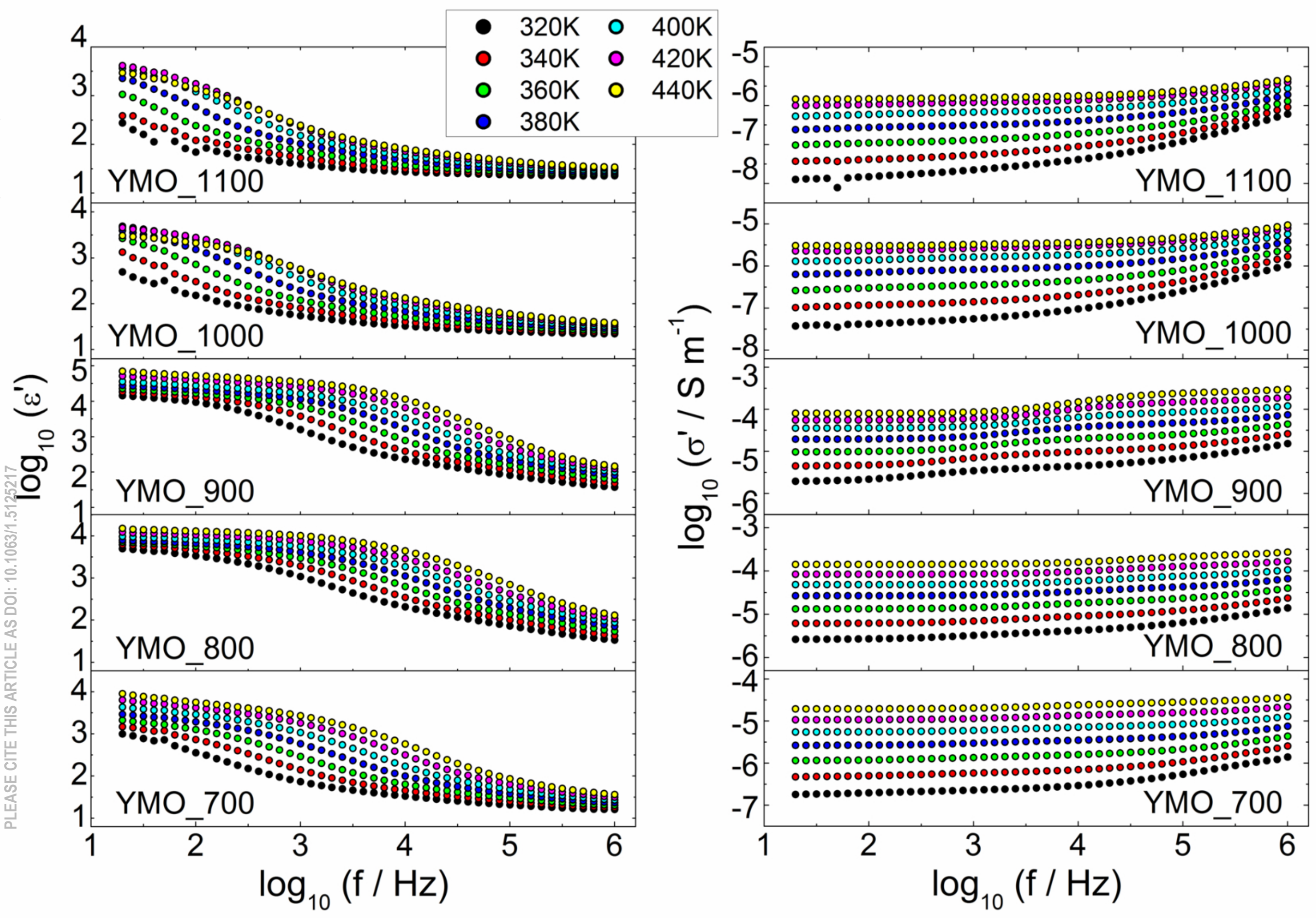




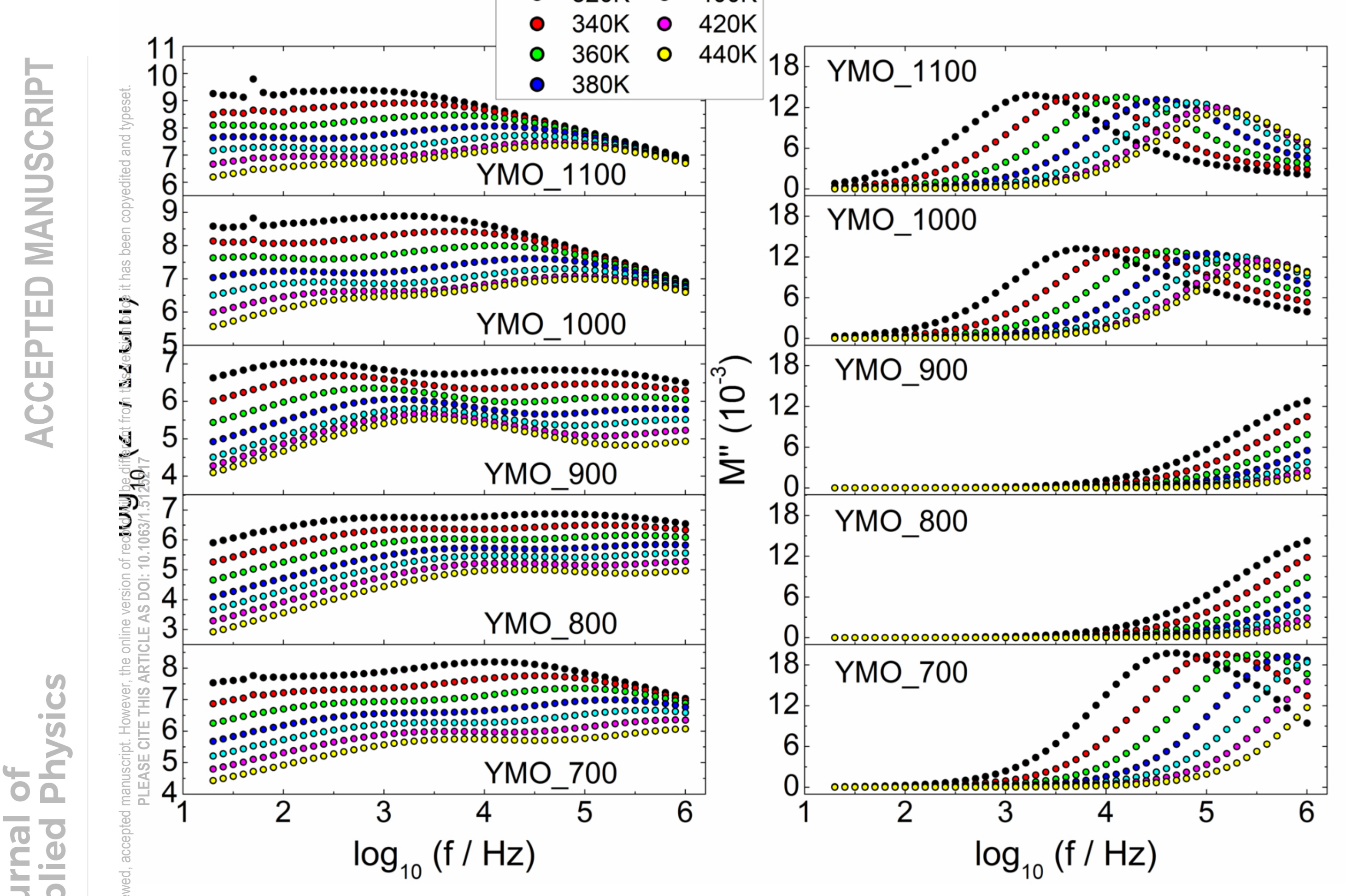



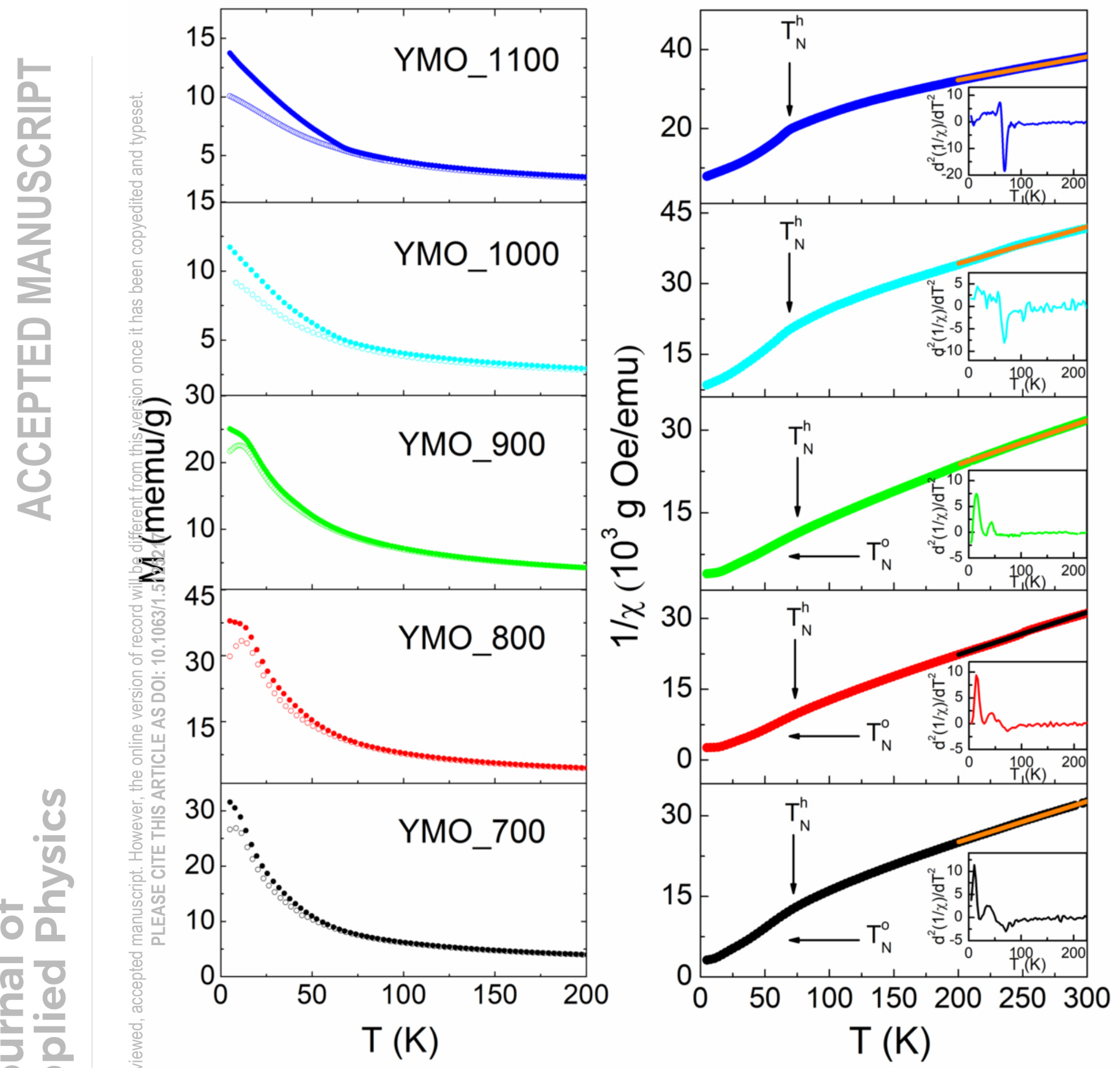


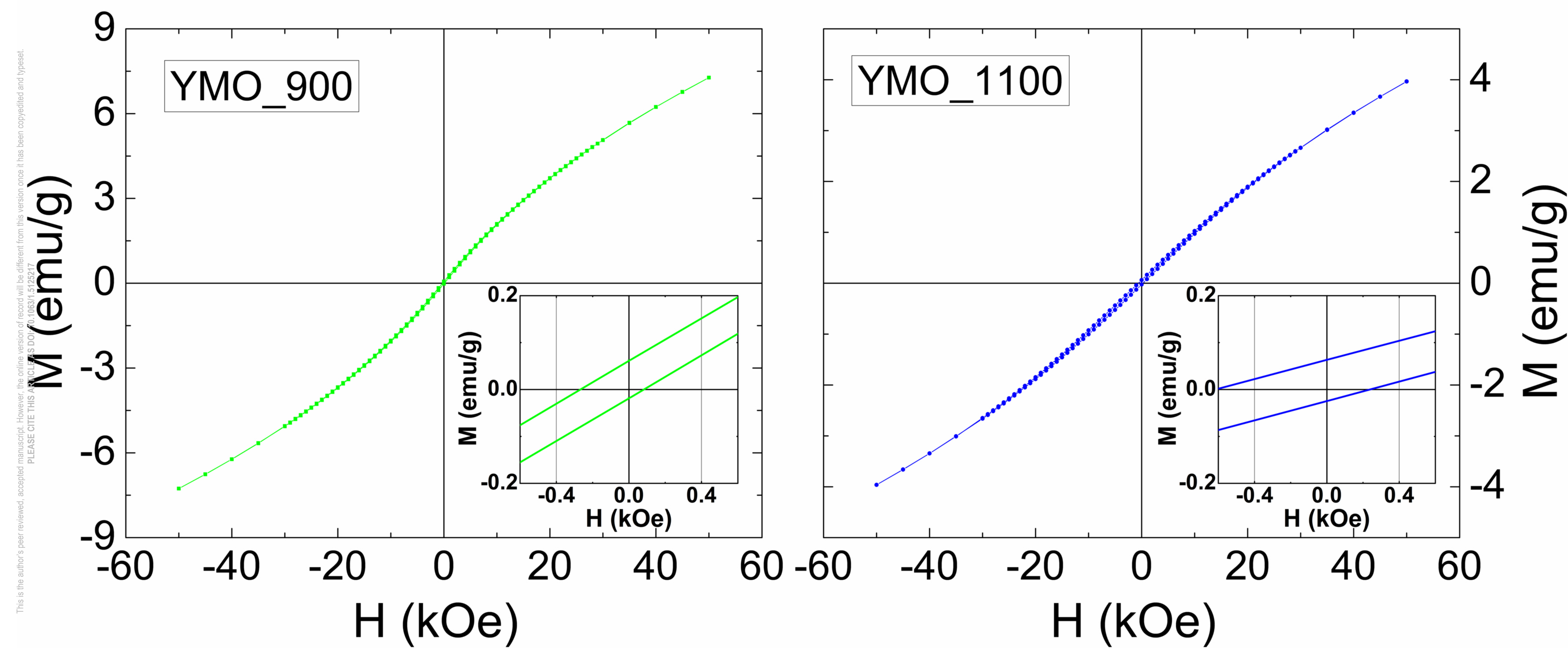

